\title{
A Reference Model System of Industrial Yeast Saccharomyces cerevisiae is needed for Development of the Next-Generation Biocatalyst toward Advanced Biofuels Production
}

\section{Z Lewis Liu ${ }^{1 *}$ and Xu Wang ${ }^{2}$}

${ }^{1}$ Bioenergy Research Unit, National Center for Agricultural Utilization Research, USDA-ARS, Peoria, USA

${ }^{2}$ Department of Applied Microbiology, Sichuan Agricultural University, Wenjiang, Sichuan 611130, China

\begin{abstract}
Diploid industrial yeast Saccharomyces cerevisiae has demonstrated distinct characteristics that different from haploid laboratory model strains. However, as a workhorse for a broad range of fermentation-based industrial applications, it was poorly characterized at the genome level. Observations on the haploid model strain performance, particularly as a host strain for new strain development, are often inconsistent with the response of the diploid industrial yeast strains. An industrial yeast model system is urgently needed for efficient development of the nextgeneration biocatalysts toward a sustainable production of advanced biofuels and chemicals.
\end{abstract}

Keywords: Industrial yeast; Lignocellulose conversion; Nextgeneration biocatalyst; Saccharomyces cerevisiae

\section{Introduction}

Industrial yeast Saccharomyces cerevisiae, commonly diploid, is a workhorse for fermentation-based industrial applications but poorly characterized at the genome level. On the other hand, haploid S. cerevisiae strain S288C is a well-known model strain widely used worldwide for life science research communities [1]. The simpler structure and well-characterized model strains are easy to use and serve as a valuable resource and reference system for human, animal, and many other life forms including microbes. For example, model strain S288C is commonly used for investigation in industrial applications, including lignocellulosic biomass conversions to chemicals and fuels. However, when it is used as a host strain for improved biocatalyst development in advanced biofuels production, the performance of the laboratory model strain derivatives is often inconsistent with that observed from strains derived from diploid host industrial yeast.

Recent studies found some significant differences in the genetic background between the haploid model strain and diploid industrial yeast strains. Genome expression of model strain S288C commonly displayed a transient response to environmental stimuli, including varied chemical challenges [2,3]. Diploid industrial yeast strains NRRL Y-12632 and Y-50049 of S. cerevisiae typically showed more persistent genome expression response to challenges of major toxic chemical compounds, such as varied furan aldehydes liberated from lignocellulosic biomass pretreatment [4-6]. Another critical issue for advanced biofuels production involves in utilization of biomass sugars embedded in cellulosic materials. Traditional yeast is unable to utilize pentose sugars (C-5) such as xylose. A significant international effort has been taken to enable its C-5 sugar utilization capability through genetic engineering. When using the haploid model strain of $S$. cerevisiae as a host, such genetic engineering transformed strains with heterologous xylose transporters showed no significant improvement with a poor growth and limited xylose uptake and utilization $[7,8]$. In contrast, using similar xylose transporter genes applying on tolerant NRRL Y-50049 as a host, a significant expression of xylose uptake and utilization was obtained from the newly derived genotypes that distinct from those observed from the haploid strains [9-12]. Mechanisms behind these observations are currently unknown. On a different aspect, the laboratory strain S288C was also found to have a slower rate of genome evolution than naturally collected yeast strains [13].

In general, diploid yeast is more robust than the haploid yeast strains. A recent genomic study suggested the industrial yeast may have more tolerant signaling pathways than the model strain [14]. Reprogrammed glycolysis and pentose phosphate pathways including cofactor regeneration balance of a tolerant strain NRRL Y-50049 in response to toxic chemical challenges has been revealed $[5,12]$. A new class of aldehyde reduction gene family was defined from a diploid type yeast strain NRRL Y-12632 [5,15-17]. At least 44 pathways were reported to be affected significantly by the chemical challenges [18]. Key regulatory elements and tolerant signaling pathways were identified for the industrial yeast that may not necessarily be observed in the laboratory model strains $[6,14]$. Development of the next-generation biocatalyst is a continued challenging effort toward a sustainable bio-based economy. Since most lab strains responded differently from the industrial yeast, over-use and over-emphasis on strain performance observed from lab strains and their derivatives can be misleading and hinder the efforts of efficient new strain development. The current haploid laboratory model strains are not suitable as a host strain in biocatalyst development for lignocellulosic biomass conversion. A well-rounded model system for the industrial yeast is urgently needed for the community to successfully address challenges involved in production of fuels and chemicals from lignocellulose materials.

Numerous industrial yeast strains have been sequenced in varied depth at the genome level including both haploid and diploid strains $[14,19]$. With recent advances on investment of diploid yeast strains,

*Corresponding author: Z Lewis Liu, Bioenergy Research Unit, National Center for Agricultural Utilization Research, USDA-ARS, Peoria, IL 61604 USA, Tel: +1 309681 6294; E-mail: ZLewis.Liu@ars.usda.gov

Received October 27, 2015; Accepted November 03, 2015; Published November 10,2015

Citation: Lewis Liu Z, Wang X (2015) A Reference Model System of Industrial Yeast Saccharomyces cerevisiae is needed for Development of the NextGeneration Biocatalyst toward Advanced Biofuels Production. J Microb Biochem Technol 7: e125. doi:10.4172/1948-5948.1000e125

Copyright: (c) 2015 Lewis Liu Z, et al. This is an open-access article distributed under the terms of the Creative Commons Attribution License, which permits unrestricted use, distribution, and reproduction in any medium, provided the original author and source are credited. 
Citation: Lewis Liu Z, Wang X (2015) A Reference Model System of Industrial Yeast Saccharomyces cerevisiae is needed for Development of the Next-Generation Biocatalyst toward Advanced Biofuels Production. J Microb Biochem Technol 7: e125. doi:10.4172/1948-5948.1000e125

Page 2 of 2

NRRL Y-12632 and Y-50049 can be potential candidate model/reference for new strain development for industrial applications. High quality resequencing of the targeted genomes and updated annotations are needed for these strains. To this end, a collaborative teamwork incorporating with systems biology is necessary to establish a comprehensive database aiming the next-generation biocatalyst development for biofuels and chemical production using lignocellulosic materials.

\section{References}

1. Cherry JM, Hong EL, Amundsen C, Balakrishnan R, Binkley G, et al. (2012) Saccharomyces Genome Database: the genomics resource of budding yeast. Nucleic Acids Res 40: D700-D705.

2. Gasch AP, Spellman PT, Kao CM, Carmel-Harel O, Eisen MB, et al. (2000) Genomic expression program in the response of yeast cells to environmental changes. Mol Biol Cell 11: 4241-4257.

3. Gasch AP, Werner-Washburne M (2002) The genomics of yeast responses to environmental stress and starvation. Funct Integr Genomics 2: 181-192.

4. Liu ZL (2006) Genomic adaptation of ethanologenic yeast to biomass conversion inhibitors. Appl Microbiol Biotechnol 73: 27-36.

5. Liu ZL, Ma M, Song M (2009) Evolutionarily engineered ethanologenic yeast detoxifies lignocellulosic biomass conversion inhibitors by reprogrammed pathways. Mol Genet Genomics 282: 233-244.

6. Ma M, Liu ZL (2010) Comparative transcriptome profiling analyses during the lag phase uncover YAP, PDR, PDR, RPN, and HSF1 as key regulatory genes in genomic adaptation to the lignocellulose derived inhibitor-stress for Saccharomyces cerevisiae. BMC Genomics 11: 660.

7. Runquist $D$, Hahn-Hägerdal $B$, Rådström $P$ (2010) Comparison of heterologous xylose transporters in recombinant Saccharomyces cerevisiae. Biotechnol Biofuels 3: 5

8. Young EA, Poucher A, Comer A, Bailey and H Alper (2011) Functional survey for heterologous sugar transport proteins, using Saccharomyces cerevisiae as a host. Appl Environ Microbiol 77: 3311-3319.

9. Ma M, Liu ZL, Moon J (2012) Genetically engineered inhibitor-tolerant Saccharomyces cerevisiae through a systems biology approach is able to competitively utilize xylose and mixed biomass sugars for anaerobic ethanol fermentation. Bioenergy Res 5: 459-469.

10. Moon J, Liu ZL, Ma M, Slininger JP (2013) New genotypes of industrial yeast Saccharomyces cerevisiae engineered with YXI and heterologous xylose transporters improve xylose utilization and ethanol production. Biocatalysis Agric. Biotechnol 2: 247-254.

11. Liu ZL, Moon J, Ma M, Slininger PJ (2015) Novel yeast strains and method for lignocellulose to ethanol production. U.S. Patent 9.

12. Liu ZL (2015) Inhibitor tolerant Saccharomyces cerevisiae strain. U.S. Utility Patent 8,999,701.

13. Ronald J, Tang H, Brem RB (2006) Genomewide evolutionary rates in laboratory and wild yeast. Genetics 174: 541-544.

14. Zhou Q, Liu ZL, Ning K, Wang A, Zeng, X, et al., (2014) Genomic and transcriptome analyses reveal that MAPK- and phosphatidylinositol-signaling pathways mediate tolerance to 5-hydroxymethyl-2-furaldehyde for industrial yeast Saccharomyces cerevisiae. Scientific Reports 4: 6556.

15. Liu ZL, Moon J, Andersh BJ, Slininger PJ, Weber S (2008) Multiple genemediated $N A D(P) H$-dependent aldehyde reduction is a mechanism of in situ detoxification of furfural and 5-hydroxymethylfurfural by Saccharomyces cerevisiae. Appl Microbiol Biotechnol 81: 743-753.

16. Liu ZL, Moon J (2009) A novel NADPH-dependent aldehyde reductase gene from Saccharomyces cerevisiae NRRL Y-12632 involved in the detoxification of aldehyde inhibitors derived from lignocellulosic biomass conversion. Gene 446: 1-10.

17. Moon J, Liu ZL (2015) Direct enzyme assay evidence confirms aldehyde reductase function of Ydr541cp and Ygl039wp from Saccharomyces cerevisiae. Yeast 32: 399-407.

18. Zhang Y, Liu ZL, Song M3 (2015) ChiNet uncovers rewired transcription subnetworks in tolerant yeast for advanced biofuels conversion. Nucleic Acids Res 43: 4393-4407.

19. Juan Lucas Argueso JL, Carazzolle MF, Mieczkowski PA Duarte FM, Netto OVC, Missawa SK, et al., (2009) Genome structure of a Saccharomyces cerevisiae strain widely used in bioethanol production. Genome Res 19: 2258 2270 . 